\title{
Dysautonomia: an Explanation for the Medically Unexplained?
}

Senthil Chandrasekaram ${ }^{1}$, Ranga H Fernando ${ }^{2}$, Vikram Aarella ${ }^{1}$, Emily T Mudenha ${ }^{1}$, Devaka JS Fernando ${ }^{1}$

Department of Endocrinology and Diabetes,King's Mill Hospital, Sherwood Forest Hospital NHS Foundation Trust, Sutton-in-Ashfield, UK

\author{
${ }^{2}$ University of Manchester, Manchester, UK
}

Received: 01/04/2016

Accepted: 06/04/2016

Published: 01/06/2016

How to cite this article: Chandrasekaram S, Fernando RHK, Aarella V, Mudenha ET, Fernando DJS. Dysautonomia: an explanation for the medically unexplained? EJCRIM 2016;3:doi:10.12890/2016_000430

Conflicts of Interests: The Authors declare that there are no competing interests.

This article is licensed under a Commons Attribution Non-Commercial 4.0 License

\section{ABSTRACT}

Objective: To identify dysautonomia as a collection of conditions with variable presentation that may be mistaken for medically unexplained symptoms.

Methods: Case series.

Results: Tilt table testing and $24 \mathrm{~h}$ electrocardiographic monitoring provided useful diagnostic tools to confirm the diagnosis.

Conclusion: A greater awareness and recognition of the disorders that result from dysautonomia and recognition of the disability that results from these disorders will improve patients' quality of life.

\section{LEARNING POINTS}

- Dysautonomia is protean in presentation and clinicians need to have a high degree of suspicion as, if diagnosed, symptomatic improvement is possible leading to improved quality of life.

- Tilt table testing and $24 \mathrm{~h}$ electrocardiographic monitoring are useful diagnostic tools.

- Greater awareness and recognition of the disorders that result from dysautonomia with appropriate use of these tests by medical professionals is necessary.

\section{KEYWORDS}

Dysautonomia, medically unexplained symptoms, postural orthostatic tachycardia syndrome (POTS).

\section{INTRODUCTION}

Patients attending specialist clinics with medically unexplained physical symptoms (MUS) account for a high proportion of frequent clinic visits, multiple investigations and health care costs, with emotional and psychological effects ${ }^{[1]}$.

MUS have been divided into four categories: 1) MUS subsequently confirmed as MUS; 2) MUS initially medically explained and subsequently confirmed as medically explained; 3) MUS initially thought to be explained but later found to be unexplained (missed unexplained); and 4) MUS initially thought to be unexplained and later found to be explained (missed explained) ${ }^{[1]}$.

Dysautonomia comprises a collection of conditions which may occur in different combinations in different individuals ${ }^{[2]}$ and include postural orthostatic tachycardia syndrome (POTS), inappropriate sinus tachycardia, vasovagal syncope, pure autonomic failu re, neurocardiogenic syncope, neurally mediated hypertension, orthostatic hypotension, orthostatic hypertension, autonomic instability, paroxysmal sympathetic hyperactivity and cerebral salt wasting syndrome ${ }^{[2]}$.

We describe a series of patients with symptoms not fitting into organ-based specialities which were incorrectly labelled as psychological in origin, who had dysautonomia, thus falling into the missed explained category. 


\section{CASE 1}

A 76-year-old woman presented with a 6-year history of light-headedness, limb numbness and collapse, which had necessitated multiple admissions to hospital. Her symptoms increased with standing and were relieved on lying down. There were no gustatory sweating or bowel and bladder symptoms. Neurological examination was normal.

A formal tilt table test showed a [systolic/diastolic blood pressure drop from 222/105 to 95/73 with symptoms. Corticotrophin stimulation test, $24 \mathrm{~h}$ ECG monitoring, blood sugar and MRI of the brain were normal. Formal post-meal assessment showed post-prandial hypotension. Octreotide improved postural hypotension. It was concluded that the patient had sympathetic vasoconstrictor response failure with sparing of parasympathetic cardiac rate control.

The patient was treated with midodrine $10 \mathrm{mg}$ three times daily, fludrocortisone $50 \mu \mathrm{g}$ daily, and ephedrine escalation therapy. She was educated about increasing fluid and salt intake, regular calf and lower limb exercise and strategies to avoid syncope.

The number and frequency of hospital admissions decreased after these interventions.

\section{CASE 2}

A 29-year-old woman with dizzy spells and falls with urinary retention was diagnosed with atonic bladder and was on long-term suprapubic catheterisation with repeated admissions for recurrent catheter-associated urinary tract infections. Her dizzy spells were related to changes in posture. There was no gustatory sweating. The patient was labelled as having Munchausen's syndrome and memos to that effect were circulated to local hospitals.

On an admission to the endocrinology ward, a significant postural drop in blood pressure was noted. The tilt test was positive with a drop in systolic/diastolic blood pressure from $103 / 47$ to 50/43. The patient's heart rate increased from 48 to 175 . Her autoantibody screens, short corticotrophin test, blood glucose, vitamin B12 and folate levels were normal.

She was treated with midodrine $10 \mathrm{mg}$ three time daily and fludrocortisone $100 \mu \mathrm{g}$ daily with symptomatic improvement.

\section{CASE 3}

A 32-year-old woman with type 1 diabetes since 1997 who was treated with an insulin pump with good HBA1c <7.5 without any obvious diabetes-related complications, felt unwell, light headed, and since February 2013 had experienced palpitations and dizzy spells which were associated with changes in posture.

The tilt table test showed a drop in heart rate from 90 to 150 bpm with symptoms. Her ECG, 24 h ECG and short tetracosactrin test were normal. Her tilt test showed a fall in blood pressure. An octreotide meal test was normal.

The patient's symptoms improved with simple measures such as ensuring adequate fluid intake and voluntary muscle contractions in the lower limbs to promote venous return when standing for prolonged periods.

\section{CASE 4}

A 17-year-old girl presented with 13 episodes of dizziness followed by collapse over a period of 3 months. There was no relationship with food either as regards timing or bowel symptoms.

The patient had signs of hypermobility syndrome with a Beighton score of 8 out of 9 . Thyroid function, corticotrophin stimulation test, thyroid antibodies, renin level, aldosterone level and renal function were normal. She had a positive tilt table test with documented hypotension and tachycardia with symptoms. The diagnosis of POTS syndrome associated with hypermobility syndrome was considered in this patient.

The patient was advised to increase her water intake to 3 litres a day. She was advised to always sit in a safe place and to voluntarily contract the muscles of her limbs to help venous return. No further hospital admissions or attendances at the emergency department were recorded.

\section{DISCUSSION}

MUS are characterized by physical symptoms where investigations and clinical examinations reveal no abnormality or an abnormality thought to be trivial or incidental[ ${ }^{[1]}$.

Our patients were misclassified because the abnormalities did not fit into existing medical models, exposing our patients, their families and doctors to feelings of frustration and helplessness ${ }^{[3]}$. Patients without a diagnosis deemed acceptable by the society they live in feel that they lack the legitimacy of being recognized as 'sick' people with 'real' illnesses ${ }^{[4]}$. The frequency of psychiatric illness is no higher in people with dysautonomia than in the general population, although they are more anxious ${ }^{[5]}$. Recent studies have suggested that in situations where the legitimacy of ambiguous illnesses is questioned, coping methods useful in other conditions may not alleviate such stress and may indeed be held responsible for causing their own distress ${ }^{[5]}$. Case 2 illustrates this in the extreme where the stigma of the label of Munchausen's syndrome caused feelings of worthlessness and shame. 


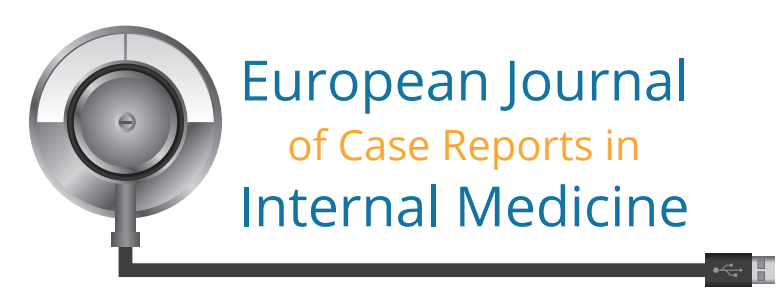

Dysautonomia is protean in presentation and clinicians need to have a high degree of suspicion. Different aspects of the syndrome may predominate in individuals ${ }^{[2]}$, leading to presentation to different organ-based clinical disciplines. If diagnosed, symptomatic improvement is possible leading to improved quality of life. Failure to recognise dysautonomia as a clinical entity delayed diagnosis and had a significant adverse impact. Tilt table testing and $24 \mathrm{~h}$ electrocardiographic monitoring are useful diagnostic tools and allow much better understanding of the pathophysiology of this condition. A greater awareness and recognition of the disorders that result from dysautonomia with appropriate use of these tests by medical professionals and recognition of the disability that results from these disorders by social services and support services is essential if people with these conditions are to receive the treatment and support they require.

\section{REFERENCES}

1. Nimnuan C, Hotopf M, Wessely S. Medically unexplained symptoms: how often and why are they missed? Q J Med 2000;93:21-28.

2. Palma JA. Not all dysautonomias are created equal. Clin Auton Res 2016;26:83.

3. Stone L. Blame, shame and hopelessness: medically unexplained symptoms and the 'heartsink' experience. Aust Fam Physician 2014;43:191-195.

4. Mclnnis OA, Matheson K, Anisman H. Living with the unexplained: coping, distress, and depression among women with chronic fatigue syndrome and/or fibromyalgia compared to an autoimmune disorder. Anxiety Stress Coping 2014;27:601-618.

5. Raj V, Haman KL, Raj SR, Byrne D, Blakely RD, Biaggioni I, et al. Psychiatric profile and attention deficits in postural tachycardia syndrome. J Neurol Neurosurg Psychiatry 2009;80:339-344. 